\title{
Sistemas computarizados para el ingreso de órdenes médicas y errores de prescripción
}

\section{Objetivo}

Identificar y cuantificar errores de prescripción causados ó exacerbados por el uso de sistemas computarizados para el ingreso de órdenes médicas (CPOE: del inglés Computerized Physician Order Entry).

Cuadro 1: sistemas computarizados para el ingreso de órdenes médicas

- Incluyen solicitud de estudios, tratamientos y otras instrucciones para el cuidado del paciente.

- Se ejecutan sin las ambigüedades de los pedidos y órdenes en papel.

- Pueden estar integrados con sistemas inteligentes para la toma de decisiones (al generar una receta, requerir un estudio ó indicar un tratamiento).

\section{Diseño}

Estudio de Evaluación cuali-cuantitativo.

\section{Lugar}

Hospital universitario de tercer nivel de Pennsylvania, EEUU, que utiliza el sistema (Eclipsys's TDS) de forma rutinaria desde el año 1997

\section{Recoleccion de Datos}

Fueron realizadas 14 entrevistas extensas a médicos de planta del hospital, cinco grupos de foco con médicos de planta moderados por un sociólogo; 14 entrevistas a expertos y directivos del hospital; seguimiento y observación personalizada durante la actividad asistencial diaria de tres médicos, nueve enfermeras y tres farmaceúticos (técnica de Shadowing ${ }^{*}$ ); y una encuesta estructurada y autoadministrada a 261 médicos del hospital que utilizan el sistema, elegidos al azar.

\section{Resultados}

Se identificaron diferentes causas de error de prescripción facilitadas por el uso de CPOE. Se las dividió en: 1) errores de información debidos a la fragmentación y a problemas de integración de los datos; 2) errores de interfaz, no asociadas con las conductas habituales de los usuarios ó a la organización. Ver cuadro 1.

Errores de Información - Fragmentación y errores de integración de los datos

\begin{tabular}{|c|c|}
\hline \multicolumn{2}{|c|}{ Errores de Información - Fragmentación y errores de integración de los datos } \\
\hline $\begin{array}{l}\text { Tipo de Error } \\
\text { Asunción de la información sobre la dosis }\end{array}$ & $\begin{array}{l}\text { Descripción } \\
\text { La información del CPOE estaba basada en información farmacéutica y no } \\
\text { sobre guías de práctica clínica. }\end{array}$ \\
\hline Falla en la discontinuación de medicación & $\begin{array}{l}\text { Duplicación de la prescripción al modificar una dosis, por no discontinuar la } \\
\text { medicación previa modificación. }\end{array}$ \\
\hline Errores en la discontinuación de medicación ligada a un procedimiento & $\begin{array}{l}\text { Si un procedimiento requería una medicación, la cancelación de este no can- } \\
\text { celaba automáticamente la prescripción medicamentosa. }\end{array}$ \\
\hline $\begin{array}{l}\text { Fallas en la discontinuación ó cancelación de medicación de "urgencia" ó } \\
\text { para "dar según necesidad" }\end{array}$ & Esta falla genera duplicaciones ó indicaciones erróneas. \\
\hline Falla en la renovación antibiótica & $\begin{array}{l}\text { La prescripción era controlada por el servicio de infectología, y al tercer día } \\
\text { de la indicación el médico debía renovar la prescripción en el sistema. La } \\
\text { falta de renovación generaba brechas sin cobertura antibiótico. }\end{array}$ \\
\hline Error en la solicitud de diluyentes & $\begin{array}{l}\text { El sistema requería indicar el diluyente para el antibiótico prescripto. Errores } \\
\text { del médico al indicar el diluyente eran controlados por personal de farmacia, } \\
\text { lo que retrasaba la indicación. }\end{array}$ \\
\hline Retraso en la información sobre alergias & $\begin{array}{l}\text { El CPOE indicaba si existía alergia a una medicación únicamente después } \\
\text { de que esta era indicada, alerta muchas veces no atendida por el médico. }\end{array}$ \\
\hline Conflictos con medicación duplicada & \\
\hline \multicolumn{2}{|c|}{ Errores de Interfaz } \\
\hline Selección de Paciente & $\begin{array}{l}\text { No todas las pantallas del CPOE tenían el nombre del paciente, la letra era } \\
\text { chica, y el orden de los pacientes era alfabético y no por servicios ó según } \\
\text { disposición hospitalaria. }\end{array}$ \\
\hline Error en la selección de medicación & $\begin{array}{l}\text { La medicación del paciente rara vez era visible en una única pantalla, por lo } \\
\text { tanto era engorrosa su visualización, lo que podía generar errores de pres- } \\
\text { cripción }\end{array}$ \\
\hline Errores en el Log in / Log out & $\begin{array}{l}\text { Era posible indicar medicaciones utilizando el usuario de otro médico que no } \\
\text { había realizado correctamente el log out. }\end{array}$ \\
\hline Falla en la prescripción postquirúrgica & $\begin{array}{l}\text { Al ingresar a una cirugía la medicación del paciente queda suspendida de- } \\
\text { biendo el médico activarla luego del procedimiento. }\end{array}$ \\
\hline Suspensión de medicación postcirugía & $\begin{array}{l}\text { El pase del paciente al quirófano suspendía las medicaciones que recibía en } \\
\text { la sala. Si no se registraba en el sistema el pase posterior a la sala, las pres- } \\
\text { cripciones no podían ser reactivadas. }\end{array}$ \\
\hline Pérdida de tiempo y datos cuando el CPOE no funciona & $\begin{array}{l}\text { Cuando el sistema no funcionaba (caída del sistema) los datos ingresados en } \\
\text { ese momento se perdían y había que reingresarlos luego. }\end{array}$ \\
\hline Envío de medicación a un cuarto erróneo cuando el sistema no funciona & $\begin{array}{l}\text { Si el pase del paciente a otra habitación no se realizaba porque el sistema } \\
\text { no funcionaba, no había alertas a la farmacia y la medicación se enviaba a } \\
\text { la vieja habitación. }\end{array}$ \\
\hline Ingresos tardíos demorados $24 \mathrm{~h}$ & $\begin{array}{l}\text { Al indicar una medicación ó prueba de laboratorio pasadas las } 0 \text { horas, el sis- } \\
\text { tema generaba la solicitud para el día siguiente, ó sea } 24 \mathrm{~h} \text { posteriores al } \\
\text { ingreso. }\end{array}$ \\
\hline Dificultades en la planificación de medicación & $\begin{array}{l}\text { Las enfermeras deben ingresar el horario en que se administró la medicación } \\
\text { en tiempo real, lo que enlentece el trabajo. }\end{array}$ \\
\hline Inflexibilidad del sistema & $\begin{array}{l}\text { No se pueden realizar solicitudes especiales para estudios ó medicación, ej: } \\
\text { ángulos especiales para una radiografía. }\end{array}$ \\
\hline
\end{tabular}

\section{Conclusiones}

Este estudio muestra que un sistema CPOE comercial, ampliamente utilizado, puede facilitar errores de medicación, y advierte a Hospitales y médicos que deben prestar atención a estos errores además de los problemas que previenen los CPOE a la hora de implementarlos. 


\section{Comentarios}

A partir del informe de la IOM publicado en el año 2000, "To Err is Human", que estima que los efectos adversos de medicación causan lesiones ó muerte a más de 98,000 personas hospitalizadas por año ${ }^{1}$ siendo los errores de prescripción la causa más frecuente ${ }^{2}$. ${ }^{7}$ distintas estrategias fueron planteadas para reducir este problema, siendo los sistemas CPOE uno de los métodos predilectos ${ }^{8}$. Múltiples estudios muestran a los CPOE como sistemas decisivos para mermar estos errores ${ }^{2,3,9,10}$ y reducir costos millonarios ${ }^{11,12}$. A partir de esta evidencia, varios estados de EEUU han creado leyes que recomiendan fuertemente la implementación de sistemas de prescripción electrónica para prevenir errores. Esto ha generado una fuerte necesidad de inversión por parte de hospitales y consultorios médicos para adquirir estos sistemas, y aún no queda claro quien debería hacerse cargo de los costos, con discusiones entre los prestadores, los aseguradores de salud y el gobierno.

En este contexto surge este estudio, evidenciando limitaciones y errores que pueden ser exacerbados por un sistema de prescripción con graves problemas de diseño. Este estudio realza la importancia de un diseño meticuloso de lo sistemas informáticos de aplicación clínica, con amplia participación de los médicos que los van a utilizar y procesos de prueba e implementación que aseguren los resultados deseados.

\section{Alejandro Mauro y Alejandro Lopez Osornio [ Informática Médica. Hospital Italiano de Buenos Aires. ]}

Mauro A, Lopez Osornio A. Sistemas computarizados para el ingreso de órdenes médicas y errores de prescripción. Evid pract ambul.2005;8:177-178. Disponible en URL: www.evidencia.org Comentado de: Koppel, R., et al., Role of computerized physician order entry systems in facilitating medication errors. Jama, 2005. 293(10): p. 1197-203. PMID 15755942 [13]

\section{Bibliografía:}

1. Lesar, T.S., B.M. Lomaestro, and H. Pohl, Medication-prescribing errors in a teaching hospital. A 9-year experience. Arch Intern Med, 1997. 157(14): p. 1569-

2. Kohn, L.T., J. Corrigan, and M.S. Donaldson, To err is human : building a safer health system. 2000, Washington, D.C.: National Academy Press. xxi, 287 p. 3. United States. Agency for Healthcare Research and Quality. and University of California San Francisco-Stanford Evidence-Based Practice Center., Making health care safer : a critical analysis of patient safety practices. Evidence report/technology assessment ; no. 43. 2001, Rockville, MD: Agency for Healthcare Research and Quality, U.S. Dept. of Health and Human Services. x, 668 p.

4. Kanjanarat, P., et al., Nature of preventable adverse drug events in hospitals: a literature review. Am J Health Syst Pharm, 2003. 60(17): p. 1750-9.

5. Leape, L.L., et al., Systems analysis of adverse drug events. ADE Prevention Study Group. Jama, 1995. 274(1): p. 35-43.

6. Bates, D.W., et al., Incidence of adverse drug events and potential adverse drug events. Implications for prevention. ADE Prevention Study Group. Jama, 1995. 274(1): p. 29-34.

7. Dean, B., et al., Causes of prescribing errors in hospital inpatients: a prospective study. Lancet, 2002. 359(9315): p. $1373-8$.

8. Institute of Medicine (U.S.). Committee on Quality of Health Care in America., Crossing the quality chasm : a new health system for the 21 st century. 2001, Washington, D.C.: National Academy Press. xx, 337 p.

9. Bates, D.W., et al., Effect of computerized physician order entry and a team intervention on prevention of serious medication errors. Jama, 1998. 280(15): $p$. $1311-6$.

10. Bates, D.W. and A.A. Gawande, Improving safety with information technology. N Engl J Med, 2003. 348(25): p. $2526-34$

11. iHealth. Frist Aide Says EMR Bill Could Pass. 2004 [cited May 1, 2004]; Available from:

http://www.ihealthbeat.org/index.cfm?Action=dspltem\&itemID=100537.

12. iHealth. Clinton Reiterates IT Stance, Details Legislation. 2004 [cited May 1, 2004; Available from:

http://www.ihealthbeat.org/index.cfm?Action=dspltem\&itemID=100529.

\section{INFO - EVIDENCIA}

\section{¿Qué es Evidencia, Actualización en la Práctica Ambulatoria?}

Una publicación independiente editada cada dos meses desde 1997 por la Fundación MF, Organización sin fines de lucro dedicada promover el desarrollo de la medicina familiar y la atención primaria de la salud.

\section{¿Cuál es el objetivo de Evidencia?}

Contribuir a la educación continua y a la actualización de los profesionales de la salud de la región en el área de la atención ambulatoria.

\section{¿Contenidos de Evidencia}

- Información independiente, actualizada y resumida en forma sencilla y en castellano sobre temas relevantes de la atención ambulatoria.

- Artículos seleccionados por su calidad y relevancia clínica, resumidos y comentados críticamente por destacados profesionales del área.

- Revisiones sobre temas clínicos, epidemiológicos, sanitarios o humanísticos, de importancia para la práctica ambulatoria.

- Notas farmacológicas para la actualización rápida de temas de terapéutica clínica.

Discusión de casos clínicos con herramientas de Medicina Basada en la Evidencia.

- Glosario de términos de epidemiología y medicina basada en la evidencia.

\section{¿Cómo es posible acceder a Evidencia?}

Por suscripción a la edición en papel, o bien por Intenet, en nuestro sitio:

http://www.evidencia.org 